\title{
ON GENERALIZATIONS OF PROJECTIVITY FOR MODULES OVER DEDEKIND DOMAINS
}

\author{
JUTTA HAUSEN \\ (Received 19 June 1980; revised 7 November 1980) \\ To my father on June 12,1981 \\ Communicated by $\mathbf{R}$. Lidl
}

\begin{abstract}
A module $M$ over a ring $R$ is $\kappa$-projective, $\kappa$ a cardinal, if $M$ is projective relative to all exact sequence of $R$-modules $0 \rightarrow A \rightarrow B \rightarrow C \rightarrow 0$ such that $C$ has a generating set of cardinality less than $\kappa$. A structure theorem for $\kappa$-projective modules over Dedekind domains is proven, and the $\kappa$-projectivity of $M$ is related to properties of $\operatorname{Ext}_{R}(M, \oplus R)$. Using results of $S$. Chase, $S$. Shelah and $P$. Eklof, the existence of non-projective $\aleph_{1}$-projective modules is shown to undecidable, while both the Continuum Hypothesis and its denial (plus Martin's Axiom) imply the existence of a reduced $\aleph_{0}$-projective $\mathbf{Z}$-module which is not free.
\end{abstract}

1980 Mathematics subject classification (Amer. Math. Soc.): primary 13 C 05, 13 C 10, 18 G 15; secondary 20 K 20, 20 K 40.

\section{Introduction}

In a recent paper, V. A. Hiremath (1978) considers finitely projective modules over a Dedekind domain $R$.

The purpose of the present article is two-fold: firstly, we wish to consider Hiremath's results on finitely projective modules in the larger setting of $\kappa$-projectivity where $\kappa$ is any infinite cardinal. Terminology is chosen such that "finitely projective" equals " $\kappa_{0}$-projective".

Secondly, we turn to the question of existence. Hiremath has shown that an $\boldsymbol{R}$-module is $\boldsymbol{N}_{0}$-projective if and only if its reduced part has this property; however, he did not address the existence problem for reduced non-projective $\boldsymbol{N}_{0}$-projective modules.

OCopyright Australian Mathematical Society 1981 
Our findings are as follows: an $R$-module $M$ is $\kappa$-projective if and only if it satisfies the condition

Every submodule $N$ of $M$ with $\kappa$-generated quotient $M / N$ (к) contains a direct summand $N^{\prime}$ of $M$ such that $M / N^{\prime}$ is projective.

(2.4). Furthermore, we show that $\aleph_{0}$-projectivity of $M$ is equivalent to $\operatorname{Ext}_{R}(M, R)$ being a torsion-free $R$-module (3.2), while for $\kappa$ uncountable and exceeding the number of prime ideals of $R,|\operatorname{spec} R|$, the $\kappa$-projectivity of $M$ is equivalent to $\operatorname{Ext}_{R}\left(M, \oplus_{\lambda} R\right)=0$ for each cardinal $\lambda<\kappa$ (4.2). Consequences are Hiremath's result that pure submodules of $\aleph_{0}$-projective modules are $\aleph_{0}$-projective (3.4), and the fact that every submodule of a $\kappa$-projective module, $\kappa$ sufficiently large, is $\kappa$-projective (4.3). Of independent interest may be our finding that, for $\kappa=\kappa_{0}$ and $M$ reduced, or $\kappa>\aleph_{0} \cdot|\operatorname{spec} R|$, condition $(\kappa)$ is equivalent to the apparently stronger condition of $M$ being $\kappa$-coseparable; that is $M$ is torsion-free, its submodules of rank less than $\kappa$ are projective, and every submodule with $\kappa$-generated quotient contains a direct summand of $M$ with $\kappa$-generated quotient (4.2).

Having related the $\kappa$-projectivity of $M$ to the structure of $\operatorname{Ext}_{R}(M, \oplus R)$, we can use results of Chase $(1963)$, Shelah $(1974,1979)$ and Eklof $(1980)$ to shed some light on the existence problem: both the Continuum Hypothesis and its denial (plus MA) imply the existence of a reduced $\aleph_{0}$-projective module $M$ which is not projective $(5.3,5.4)$. Whether the existence of such $M$ can be derived from within our standard set theoretical framework (Zermelo-Fraenkel plus Axiom of Choice) is an unsolved problem.

For $\kappa>\kappa_{0}$ and $R$ a countable Dedekind domain which is not a field, every $\kappa$-projective $R$-module is projective if one assumes the Axiom of Constructibility; assuming Martin's Axiom and $2^{\kappa_{0}}>\aleph_{1}$ instead, there exist $\aleph_{1}$-projective Z-modules which are not free $(5.1,5.2)$. Thus, for $\kappa=\kappa_{1}$, the existence of non-projective $\kappa$-projective modules is independent of and consistent with our standard set theory. Whether this is true for any cardinal $\kappa>\aleph_{0}$ is not known to us.

Throughout, all modules are unital left modules and $\mathbf{Z}$ denotes the ring of integers.

\section{2. $\kappa$-Projective modules}

Let $\kappa$ be a cardinal. Following Eklof and Huber (1979), p. 449, we call a module $M$ over a ring $R \kappa$-generated if it has an $R$-generating set of cardinality strictly less than $\kappa$. And $M$ is $\kappa$-projective if $M$ is projective relative to all exact 
sequences of $R$-modules $0 \rightarrow A \rightarrow B \rightarrow C \rightarrow 0$ with $C \kappa$-generated. Clearly,

(2.1) Every $\kappa$-projective module is $\lambda$-projective, for any cardinal $\lambda$ less than $\kappa$.

A consequence of Anderson and Fuller (1974), p. 186, 16.10, is

(2.2) Direct sums and direct summands of $\kappa$-projective modules are $\kappa$-projective.

These and other properties of $\kappa$-projective modules hold in much more general context: if $\underline{C}$ is a class of $R$-modules which is closed with respect to isomorphic copies, define a module $M$ to be $\underline{C}$-projective if $M$ is projective relative to all short exact sequences

$$
0 \rightarrow A \rightarrow B \rightarrow C \rightarrow 0
$$

of $R$-modules with $C$ belonging to $\underline{C}$. Standard arguments prove that, if $\underline{C}$ is closed with respect to homomorphic images, then $\underline{C}$-projective modules belonging to $\underline{C}$ are projective. Hence

(2.3) Every $\kappa$-projective $\kappa$-generated module is projective.

From now on assume that $R$ is Dedekind domain. Thus, by Cartan and Eilenberg (1956), p. 14, 5.4, submodules of projective $R$-modules are projective. It follows from Kaplanky (1952), p. 333, that submodules of $\kappa$-generated $R$-modules, $\kappa$ infinite, are $\kappa$-generated.

The following result, again, could have been formulated for $\underline{C}$-projective modules; for this we would need that $\underline{C}$ is closed with respect to submodules and epimorphic images and contains enough projectives. Following Anderson and Fuller (1974), we call $M B$-projective if $M$ has the projective property relative to all exact sequences of $R$-modules $0 \rightarrow A \rightarrow B \rightarrow C \rightarrow 0$.

(2.4) Theorem. Let $R$ be a Dedekind domain and let $\kappa$ be an infinite cardinal. The following properties of the $R$-module $M$ are equivalent.

(i) $M$ is к-projective.

(ii) For every cardinal $\lambda<\kappa, M$ is $\bigoplus_{\lambda} R$-projective.

(iii) Every submodule $N$ of $M$ with $\kappa$-generated quotient $M / N$ contains a direct summand $N^{\prime}$ of $M$ such that $M / N^{\prime}$ is $\kappa$-generated and projective.

(iv) Every submodule $N$ of $M$ with $\kappa$-generated quotient $M / N$ contains a direct summand $N^{\prime}$ of $M$ such that $M / N^{\prime}$ is projective.

Proof. (ii) $\Rightarrow$ (iii): If $N<M$ with $M / N$ k-generated, there exists a cardinal $\lambda<\kappa$ and an epimorphism $\alpha: F \rightarrow M / N$ where $F=\bigoplus_{\lambda} R$. Thus for $\zeta: M \rightarrow$ $M / N$ the natural map, $\zeta=\alpha \circ \psi$ for some $\psi: M \rightarrow F$. Hence $\psi(M)<F$ is $\kappa$-generated and projective implying $M=\operatorname{ker} \psi \oplus Q, Q \simeq \psi(M)$ as desired.

(iv) $\Rightarrow$ (i): Consider a diagram

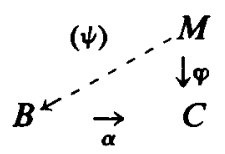


with $C$ k-generated and $\alpha$ epic. Then $\varphi(M) \simeq M / \operatorname{ker} \varphi$ is $\kappa$-generated, so that $M=K \oplus Q$ with $K \leqslant \operatorname{ker} \varphi$ and $Q$ projective. Thus, there exists $\psi^{\prime}: Q \rightarrow B$ such that $\alpha \circ \psi^{\prime}=\varphi \mid Q$. Extend $\psi^{\prime}$ to a map $\psi: M \rightarrow B$ by defining $\psi(K)=0$.

Let $Q(R)$ denote the quotient field of $R$ and suppose $R \neq Q(R)$. For $P$ a maximal ideal of $R$, let $Z\left(P^{\infty}\right)$ denote the $P$-primary component of $Q(R) / R$. Then $Z\left(P^{\infty}\right)$ is $\aleph_{1}$-generated, every divisible $R$-module is a direct sum of copies of $Q(R)$ and $Z\left(P^{\infty}\right)$ for various maximal ideals $P$, and every $R$-module is a direct sum of a divisible and a reduced submodule; see Kaplansky (1952).

(2.5) Lemma. Let $R$ be a Dedekind domain which is not a field, let $\kappa$ be an infinite cardinal and let $M$ be a $\kappa$-projective $R$-module. Then:

(i) If $\kappa>\kappa_{0}$ then $M$ is reduced.

(ii) If $M$ is reduced then $M$ is torsion-free and every submodule of countable rank of $M$ is projective.

Proof. For (i), assume $\kappa>\aleph_{0}$ and not every $\kappa$-projective module is reduced. Then, by (2.2), there exists a $\kappa$-projective divisible module $D$ and an epimorphism $\varphi: D \rightarrow Z\left(P^{\infty}\right)$ where $P$ is some maximal ideal of $R$. If $F$ is free of countable rank then $\operatorname{Hom}_{R}(D, F)=0$. Hence $\varphi$ cannot be factored through $F$-contradiction.

For part (ii), observe that, by Hiremath (1978), p. 332, Theorem 8, a reduced $\kappa_{0}$-projective module is torsionless which as is well known implies the conclusion of (ii).

For $R$ a Dedekind domain, the relevant properties of Ext ${ }_{R}$ are identical with those of Ext $_{\mathrm{z}}$. Since $R$ is commutative, Ext ${ }_{R}$ is an $R$-module; see Cartan and Eilenberg (1956) for details. No confusion should arise by writing Ext instead of $\mathrm{Ext}_{R}$.

(2.6) Lemma. Let $M$ be a module over a Dedekind domain and let $\kappa$ be a cardinal. If $M$ is $\kappa$-projective then, for each cardinal $\lambda<\kappa$, the $R$-module $\operatorname{Ext}\left(M, \bigoplus_{\lambda} R\right)$ is torsion free.

Proof. Let $\lambda<\kappa$, let $F=\bigoplus_{\lambda} R$, and let $0 \neq r \in R$. Since $0 \rightarrow$ $F \stackrel{r \cdot 1}{\rightarrow} F \stackrel{\alpha}{\rightarrow} F / r F \rightarrow 0$ is exact, so is the induced sequence

$$
\operatorname{Hom}(M, F) \stackrel{\alpha^{\prime}}{\rightarrow} \operatorname{Hom}(M, F / r F) \stackrel{\gamma}{\rightarrow} \operatorname{Ext}(M, F) \stackrel{\beta}{\rightarrow} \operatorname{Ext}(M, F),
$$

where $\beta=r \cdot 1_{\operatorname{Ext}(M, F)}$ is the multiplication by $r . M$ being $\kappa$-projective implies that $\alpha^{\prime}$ is surjective. Hence $\gamma=0$ proving $\operatorname{ker}(\beta)=0$ as desired. 


\section{The countable case}

Some terminology, motivated by Griffith (1970), p. 133, will be convenient. Again, $\kappa$ is a cardinal and $R$ is a Dedekind domain.

(3.1) Definition. An $R$-module $M$ is $\kappa$-coseparable if (i) $M$ is torsion-free; (ii) every submodule of $M$ of rank less than $\kappa$ is projective; and (iii) every submodule $N$ of $M$ with $\kappa$-generated quotient $M / N$ contains a direct summand $N^{\prime}$ of $M$ with $\kappa$-generated quotient $M / N^{\prime}$.

Hiremath (1978), p. 331, Proposition 6, has shown that a module over a Dedekind domain is $\kappa_{0}$-projective if and only if its reduced part has this property. We have the following characterization which holds with and without the parenthetical statement in (ii).

(3.2) Theorem. Let $M$ be a module over a Dedekind domain $R$. The following conditions are equivalent.

(i) $M$ is $\aleph_{0}$-projective.

(ii) Every submodule $N$ of $M$ such that $M / N$ is finitely generated contains a direct summand $N^{\prime}$ of $M$ such that $M / N^{\prime}$ is projective (and finitely generated).

(iii) $M=D \oplus N$ where $D$ is a divisible and $N$ is an $\kappa_{0}$-coseparable $R$-module.

(iv) The $R$-module $\operatorname{Ext}(M, R)$ is torsion-free.

Remark. By Theorem 2.3, p. 655, of Griffith (1968), (i)-(iv) of (3.2) are equivalent with the condition that $M=D \oplus N$ with $D$ divisible and $N$ both $\aleph_{0}$-coseparable and separable. Griffith's proof however depends on the machinery of Chase (1962), Theorem 4.2.

Proof of (3.2). (i) $\Leftrightarrow$ (ii): (2.4).

(i) $\Leftrightarrow$ (iii): Hiremath (1978), p. 331, Proposition 6, together with (2.2), (2.4), and (2.5) above.

(i) $\Rightarrow$ (iv): (2.6).

(iv) $\Rightarrow$ (i): Let $M=D \oplus N$ with $D$ divisible and $N$ reduced. If $P$ is a maximal ideal of $R$ then $\operatorname{Ext}\left(R / P^{n}, R\right)$ is a non-zero torsion module for each positive integer $n$. Hence $N$ cannot have cyclic primary summands $\neq 0$ so that, by Kaplansky (1952), p. 336, Theorem 9, $N$ must be torsion-free. By (2.4) it suffices to show that $N$ is $F$-projective for each free module $F$ of finite rank. Let $\alpha$ : $F \rightarrow C$ and $\varphi: N \rightarrow C$ be homomorphisms with $\alpha$ epic. Since $C$ is finitely generated, by Kaplansky (1952), $C=B \oplus Q$ with $B$ bounded and $Q$ projective. 
It follows that there exists a decomposition

$$
N=N^{\prime} \oplus Q^{\prime}, \quad N^{\prime}=\varphi^{-1}(B), Q^{\prime} \text { projective. }
$$

Consequently, for some homomorphism $\psi^{\prime}: Q^{\prime} \rightarrow F, \alpha \circ \psi^{\prime}=\varphi \mid Q^{\prime}$. The proof will be completed once we show the existence of a homomorphism $\psi^{\prime \prime}: N^{\prime} \rightarrow F$ such that $\alpha \circ \psi^{\prime \prime}=\varphi \mid N^{\prime}$. Let $K$ denote the kernel of $\alpha$. The exactness of $0 \rightarrow K \rightarrow F \stackrel{\alpha}{\rightarrow} C \rightarrow 0$ implies the exactness of

$$
\operatorname{Hom}\left(N^{\prime}, F\right) \stackrel{\alpha^{*}}{\rightarrow} \operatorname{Hom}\left(N^{\prime}, C\right) \stackrel{\gamma}{\rightarrow} \operatorname{Ext}\left(N^{\prime}, K\right) \rightarrow \operatorname{Ext}\left(N^{\prime}, F\right),
$$

where $\alpha^{*}$ is the map induced by $\alpha$. Since $\operatorname{Ext}\left(N^{\prime}, F\right)$ is a direct summand of

$$
\operatorname{Ext}(N, F)=\operatorname{Ext}(N, \underset{\text { fin }}{\oplus} R) \simeq \bigoplus \operatorname{Ext}(N, R),
$$

which is torsion-free, the image of $\gamma$ is a pure submodule of $\operatorname{Ext}\left(N^{\prime}, K\right)$; but, by Cartan and Eilenberg (1956), p. 135, 5.3, $N^{\prime}$ torsion-free $i m p l i e s \operatorname{Ext}\left(N^{\prime}, K\right)$ divisible. Hence $\gamma\left(\operatorname{Hom}\left(N^{\prime}, C\right)\right)$ is divisible. Regarding $\operatorname{Hom}\left(N^{\prime}, B\right)$ in a natural way as a submodule of $\operatorname{Hom}\left(N^{\prime}, C\right)$, we have $\varphi \mid N^{\prime} \in \operatorname{Hom}\left(N^{\prime}, B\right)$ and $\gamma\left(\operatorname{Hom}\left(N^{\prime}, B\right)\right)=0$ since $B$ is bounded. It follows that $\varphi \mid N^{\prime}$ belongs to the irnage of $\alpha^{*}$ and the proof is completed.

We list a consequence which may be of interest in the construction of non-projective $\aleph_{0}$-projective reduced modules.

(3.3) Corollary. $A$ reduced module $M$ over a Dedekind domain is $\aleph_{0}$-coseparable if and only if every submodule $N$ of $M$ with finitely generated quotient $M / N$ contains a direct summand $N^{\prime}$ of $M$ such that $M / N^{\prime}$ is projective.

Using standard homological arguments, the equivalence of (i) and (iv) in (3.2) proves the following result due to Hiremath.

(3.4) CoRollary (Hiremath (1978)). Pure submodules of $\aleph_{0}$-projective modules over Dedekind domains are $\aleph_{0}$-projective.

If $M$ is an $R$-module such that $\operatorname{Ext}(M, R)=0$ then $M$ is said to be a Whitehead module. By (3.2), every Whitehead module is $\aleph_{0}$-projective. Assume that $R$ is a countable Dedekind domain which is not a field. Then every Whitehead $R$-module is reduced (see Eklof (1980), p. 85, 9.1) and torsion-free (2.5). Thus, using (3.2) and (2.5), we have derived

(3.5) COROLLARY. If $R$ is a countable Dedekind domain which is not a field, then every Whitehead $R$-module is reduced, $\boldsymbol{N}_{0}$-projective and $\boldsymbol{N}_{0}$-coseparable. 


\section{The uncountable case}

Let, again, $R$ be a Dedekind domain, and let $\operatorname{spec} R$ denote the set of prime ideals of $R$. If $\kappa_{0}=\kappa_{0} \cdot|\operatorname{spec} R|$, then the quotient field of $R$ has an $R$-generating set of cardinality $\kappa_{0}$; furthermore, for every cardinal $\kappa>\kappa_{0}$, every torsionfree $R$-module of rank less than $\kappa$ is $\kappa$-generated.

The proof of the following result is modeled after Griffith (1970), p. 132, proof of Theorem 190.

(4.1) Lemma. Let $M$ be a $\kappa$-projective $R$-module where $\kappa>\aleph_{0} \cdot|\operatorname{spec} R|$. Then for all ordinals $\lambda<\kappa, \operatorname{Ext}\left(M, \oplus_{\lambda} R\right)=0$.

Proof. Fix $\lambda<\kappa$, let $F=\bigoplus_{\lambda} R$, and consider an exact sequence

$$
0 \rightarrow F \stackrel{i}{\rightarrow} X \stackrel{j}{\rightarrow} M \rightarrow 0,
$$

where $i$ is the inclusion map. By (2.5), $M$ is torsion-free. Let $H$ be an $F$-high submodule of $X$. One verifies that $H$ is pure in $X$ and $X /(F \oplus H)$ is torsion (see Feigelstock (1977), p. 259, Lemma 9). Hence $X / H$ and $(F \oplus H) / H \simeq F$ have equal rank $\lambda<\kappa$ so that, by the remark above, $X / H$ is $\kappa$-generated. Then so is

$$
M / j(H)=\frac{j(X)}{j(F \oplus H)} \simeq \frac{X}{F \oplus H},
$$

and because of (2.4), $M=K \oplus B$ where $K \subseteq j(H)$ and $B$ is $\kappa$-generated and projective. If $C=j^{-1}(B)$ and $E=j^{-1}(K) \cap H$, one verifies as in Griffith (1970), p. 132, that $X=C \oplus E$. By construction, $B=j(C) \simeq C / F$ is projective, hence $C=F \oplus B^{\prime}$ and the given sequence splits.

The following theorem is the counterpart to (3.2). The equivalence of (iii) and (iv) is due to Griffith. Again, the equivalence of (ii) and (iii) may facilitate the construction of non-projective $\kappa$-coseparable modules. The theorem is valid both with and without the parenthetical statement in (ii).

(4.2) THEOREM. Let $R$ be a Dedekind domain and let $\kappa$ be an uncountable cardinal which is larger than the number of prime ideals of $R$. Then the following properties of the $R$-module $M$ are equivalent.

(i) $M$ is $\kappa$-projective.

(ii) Every submodule $N$ of $M$ with $\kappa$-generated quotient $M / N$ contains a direct summand $N^{\prime}$ of $M$ such that $M / N^{\prime}$ is projective (and $\kappa$-generated).

(iii) $M$ is $\kappa$-coseparable.

(iv) For every cardinal $\lambda<\kappa, \operatorname{Ext}\left(M, \oplus_{\lambda} R\right)=0$. 
Proof. (i) $\Leftrightarrow$ (ii): (2.4).

(i) $\Rightarrow$ (iv): (4.1).

(iv) $\Rightarrow$ (i): By (2.4), it suffices to show that $M$ is $F$-projective for every free $R$-module $F$ of infinite rank $\lambda<\kappa$. Every submodule $S$ of such $F$ is isomorphic to a direct summand of $F$. Consequently, $\operatorname{Ext}(M, S)=0$ and the exactness of a sequence $0 \rightarrow S \rightarrow F \stackrel{\alpha}{\rightarrow} C \rightarrow 0$ implies the exactness of $\operatorname{Hom}(M, F) \stackrel{\alpha^{\prime}}{\rightarrow} \operatorname{Hom}(M, C) \rightarrow 0$ where $\alpha^{\prime}$ is the induced map.

This completes the proof of the equivalence of (i), (ii), and (iv). Since, for every submodule $S$ of $M, \operatorname{Ext}(M, F) \rightarrow \operatorname{Ext}(S, F) \rightarrow 0$ is exact, it follows that submodules of $\kappa$-projective modules are $\kappa$-projective as stated in the first corollary below. In order to derive (iii) from the equivalent properties (i), (ii), (iv), it suffices to show that every $\kappa$-projective module $M$ is torsion-free and its submodules of rank less than $\kappa$ are projective. The first assertion follows from (2.5), and the second one from (2.3) and the fact that such submodules are both $\kappa$-generated and $\kappa$-projective. The final implication from (iii) to (ii) is trivial.

(4.3) Corollary. Let $R$ be a Dedekind domain and let $\kappa>\kappa_{0} \cdot|\operatorname{spec} R|$. Then submodules of $\kappa$-projective $R$-modules are $\kappa$-projective.

(4.4) Corollary. For $\kappa$ an uncountable cardinal, every $\kappa$-projective module over a countable Dedekind domain is a Whitehead module.

\section{Existence problems}

A Whitehead Z-module is called a Whitehead group. The existence of nonfree Whitehead groups was a long standing problem that was solved by Saharon Shelah (1974) in a most surprising way: two axioms, each independent of and consistent with our standard logical framework, the Zermelo-Fraenkel set theory, $\mathrm{ZF}$, plus the Axiom of Choice, $\mathrm{AC}$, lead to contradictory answers when added to $\mathrm{ZF}+\mathrm{AC}$. One of these is the Axiom of Constructibility, $V=L$; the other one is Martin's Axiom, MA, together with the denial of the Continuum Hypothesis, $\mathbf{C H}$.

Assuming Martin's Axiom and $2^{*_{0}}>\aleph_{1}$, Shelah (1974) established the existence of a non-free Whitehead group of cardinality $\aleph_{1}$; furthermore, by Shelah (1979), p. $312,1.1$, an abelian group $A$ of cardinality $\aleph_{1}$ is a Whitehead group if and only if $\operatorname{Ext}\left(A, \oplus_{\kappa_{0}} Z\right)=0$. Thus (4.2) implies

(5.1) THEOREM (MA $+\neg \mathrm{CH})$. There exists an abelian group of cardinality $\boldsymbol{*}_{1}$ which is $\aleph_{1}$-projective but not free. 
In contrast to this, Eklof (1980), p. 86, 9.2, has shown that, assuming the Axiom of Constructibility, every Whitehead module over a countable Dedekind domain is projective. Thus observing (4.4),

(5.2) Theorem $(V=L)$. For $\kappa$ an uncountable cardinal, every $\kappa$-projective module over a countable Dedekind domain is projective.

It follows that the existence of non-projective $\aleph_{1}$-projective modules is independent of and consistent with our standard set theory.

For $\boldsymbol{\aleph}_{0}$-projective modules, the situation is different. By Hiremath (1978), every divisible $R$-module is $\aleph_{0}$-projective. This raises the question whether there exist reduced $\boldsymbol{\kappa}_{0}$-projective modules which are not projective. Assuming the Continuum Hypothesis, Chase (1963), p. 191, 4.4, has given an affirmative answer: there exists a reduced non-free abelian group $A$ of cardinality $\aleph_{1}$ such that $\operatorname{Ext}(A, Z)$ is torsion-free. Thus, (3.2) implies

(5.3) TheOREM (CH). There exists a reduced $\aleph_{0}$-projective abelian group of cardinality $\aleph_{1}$ which is not free.

Let $R$ be a countable Dedekind domain which is not a field. Eklof (1980), p. 86, 9.2, has established the existence of a non-projective Whitehead $R$-module, again assuming MA and denying $\mathrm{CH}$. Combining this with (3.5) we obtain

(5.4) TheOREM (MA $+\neg \mathrm{CH}$ ). If $R$ is a countable Dedekind domain which is not a field, then there exists a reduced $\boldsymbol{\kappa}_{0}$-projective $R$-module which is not projective.

Thus, both the Continuum Hypothesis (which is a consequence of $\mathrm{ZF}$ and $V=L$ ) and its denial (plus MA) imply the existence of non-projective $\aleph_{0}$-projective reduced modules. Whether such modules must exist without augmenting our standard set theoretical framework is an unsolved problem.

\section{References}

F. W. Anderson and K. R. Fuller (1974), Rings and categories of modules (Springer-Verlag, New York).

H. Cartan and S. Eilenberg (1956), Homological algebra (Princeton University Press, Princeton, New Jersey).

S. Chase (1962), 'Locally free modules and a problem of Whitehead', Illinois J. Math. 6, 682-699.

S. Chase (1963), 'On group extensions and a problem of J. H. C. Whitehead', Topics in abelian groups, edited by J. M. Irwin and E. A. Walker, pp. 173-193 (Scott, Foresman, Chicago). 
P. C. Eklof (1980), 'Set theoretic methods in homological algebra and abelian groups', Proceedings, 18 e session du Séminaire de mathématiques supérieures, Groupes abéliens, modules et sujets connexes, pp. 7-117. (Les Presses de l'Université de Montréal, Montréal).

P. C. Eklof and M. Huber (1979), 'Abelian group extensions and the axiom of constructibility', Comment. Math. Helv. 54, 440-457.

S. Feigelstock (1977), 'On modules over Dedekind rings', Acta. Sci. Math. (Szeged) 39, 225-263.

P. Griffith (1968), 'Separability of torsion free groups and a problem of J. H. C. Whitehead', Illinois J. Math. 12, 654-659.

P. Griffith (1970), Infinite abelian group theory (University of Chicago Press, Chicago).

V. A. Hiremath (1978), 'Finitely projective modules over a Dedekind domain', J. Austral. Math. Soc. Ser. A 26, 330-336.

I. Kaplansky (1952), 'Modules over Dedekind rings and valuation rings', Trans. Amer. Math. Soc. 71, 327-340.

R. J. Nunke (1959), 'Modules of extensions over Dedekind rings', Illinois J. Math. 3, 222-241.

S. Shelah (1974), "Infinite abelian groups, Whitehead problem and some constructions", Israel $J$. Math. 18, 243-356.

S. Shelah (1979), "On uncountable abelian groups", Israel J. Math. 32, 311-330.

Department of Mathematics

University of Houston, Central Campus

Houston, Texas 77004

U.S.A. 